\title{
Labyrinthe
}

19 | 2004 (3)

Le Bel Aujourd'hui

\section{La poétique de l'émerveillement dans le Roland amoureux de Boiardo}

\section{Pascaline Nicou}

\section{(2) OpenEdition \\ 1 Journals}

Édition électronique

URL : http://journals.openedition.org/labyrinthe/253

DOI : $10.4000 /$ labyrinthe.253

ISSN : 1950-6031

Éditeur

Hermann

\section{Édition imprimée}

Date de publication : 15 décembre 2004

Pagination : 139-143

\section{Référence électronique}

Pascaline Nicou, « La poétique de l'émerveillement dans le Roland amoureux de Boiardo », Labyrinthe [En ligne], 19 | 2004 (3), mis en ligne le 19 juin 2008, consulté le 02 mai 2019. URL : http:// journals.openedition.org/labyrinthe/253; DOI : 10.4000/labyrinthe.253 


\title{
LA POÉTIQUE DE L'ÉMERVEILLEMENT DANS LE ROLAND AMOUREUX DE BOIARDO*
}

\author{
Pascaline NiCOU \\ nicou.pascaline@wanadoo.fr
}

Le Roland amoureux est un poème chevaleresque écrit pendant une trentaine d'années par le conte Matteo Maria Boiardo, seigneur de Scandiano, dans le contexte de la Ferrare renaissante des ducs d'Este, où primait l'influence de la culture française médiévale et de la civilisation courtoise. Pour Boiardo, homme de cour, il s'agissait d'écrire un poème chevaleresque dans le respect le plus strict du genre tout en le rehaussant sur le plan de l'écriture et de l'invention narrative, pour plaire au duc Borso d'Este, grand amateur des histoires chevaleresques mais aussi à son successeur Hercule d'Este, promoteur de la culture classique, et certainement pour divertir un plus large public. Le poème de Boiardo, très apprécié à son époque et attendu avec impatience par la cour, n'a pas été bien reçu au siècle suivant, occulté par le succès du Roland furieux de l'Arioste, et jugé comme «rude» en raison de son écriture dialectale et hybride, mélangeant les tons trivial et élevé. Il n'a jamais été traduit en France, si ce n'est dans des traductions-adaptations en prose, du XVI ${ }^{e}$ au XVII ${ }^{e}$ siècle. Il a même été réécrit au XVI ${ }^{e}$ siècle par Francesco Berni, dans une version normalisée qui enlevait tout ce qui faisait le propre de l'original, et le texte premier de Boiardo n'a été redécouvert qu'au XIX ${ }^{\mathrm{e}}$ siècle.

Notre intention est de montrer en quoi l'écriture singulière du Roland amoureux, laquelle lui a valu toutes ces mésaventures, est régie par un principe poétique fort, basé sur l'émerveillement. Notre thèse est que cet émerveillement est suscité par une oscillation constante, à

\footnotetext{
* Thèse sous la direction du professeur Frank La Brasca, université de Tours : «La transmission du merveilleux dans le Roland amoureux de Boiardo. Une poétique de l'émerveillement. » Soutenance prévue en décembre 2004.
} 
tous les niveaux du récit, entre une familiarité, une répétitivité ou banalité d'un côté, et une distanciation ludique, un dépaysement fondé sur des trouvailles inédites et étonnantes, de l'autre.

Boiardo reprend les deux grandes traditions qui imprègnent son époque et sa région, les romans de types carolingiens (les chansons de geste comme L'Entrée d'Espagne, en langue franco-vénitienne, mais aussi les poèmes italiens d'Andrea da Barberino), au style humble, et les romans arthuriens plus élevés tels Lancelot, Tristan, Guiron le courtois, suivis de leurs vulgarisations italiennes, comme la Tavola Ritonda. Dans le Roland amoureux, en effet, le paladin Roland, principal chevalier de la cour de Charlemagne, tombe amoureux de la magicienne Angélique et son amour lui fait remporter un certain nombre d'épreuves, comme Lancelot du lac dans le roman éponyme. Ces deux types de romans fournissent des motifs de merveilleux comme les duels extraordinaires, où les héros déploient une force surhumaine, et les espaces enchantés peuplés de figures monstrueuses que les héros rencontrent. Si Boiardo répète ces motifs tout au long du récit, ce qui crée une habitude ou familiarité, il les rend encore plus surprenants, ce qui suscite un effet d'émerveillement. Par exemple, le motif du saut du chevalier s'illustre dans un bond prodigieux où le héros n'a qu'à prendre du recul pour traverser un fleuve, ou encore le motif du combat avec un monstre devient une scène d'une violence expressive extraordinaire puisque l'un des héros, Mandricard, tombe dans un ravin et fait exploser les yeux du monstre en l'écrasant de son poids. Rien de tel dans la tradition précédente des romans arthuriens ou même des cantari, chanteurs populaires qui reprenaient oralement un certain nombre de ces récits. De la même façon, le motif topique des armes brisées au cours du combat se transforme en une vision surréelle où les armes, tant elles ont été réduites en poussière, disparaissent de la surface de la terre. De plus, la narration, basée sur le principe de l'entrelacement issu des romans médiévaux français, crée sans cesse le suspens et la surprise: en effet, le récit s'interrompt tout le temps au beau milieu d'un chant, d'un duel, au plus fort de la tension, pour reprendre le fil d'une aventure merveilleuse et vice versa; celle-ci s'arrête et l'on revient au combat.

Mais l'innovation principale de notre poète, outre l'insertion de cette structure narrative élevée et de ces images nouvelles, consiste à introduire, au sein d'un récit à la rhétorique humble, des éléments de 
la tradition classique, gréco-latine (Virgile, Ovide, Stace) et de la tradition lyrique florentine (Boccace, Pétrarque, Dante). Cette opposition entre deux types de registres crée une sensation d'étonnement chez le lecteur qui en perçoit les échos. La matière merveilleuse antérieure (mythologique, épique, arthurienne, lyrique), matière intertextuelle privilégiée, est reprise de façon singulière par Boiardo qui se livre à ce que nous appelons une traduction ludique. Il s'agit en effet d'une réécriture qui suit un double mouvement, de rabaissement comique ou farcesque, parodique (au sens étymologique du terme de para et de ôdè, ce qui signifie chanter à côté, en contrepoint, et donc transposer une mélodie, comme le relève Genette dans Palimpsestes) et d'amplification élevée, d'emphase extraordinaire. Le merveilleux épique des combats extraordinaires est tantôt repris de façon sérieuse et solennelle, par exemple lorsque Roland fend les ennemis «comme un fleuve ouvre la mer », tantôt de manière parodique, quand Roland fuit le combat et prie pour la perte des siens, afin d'arriver en triomphateur et de remporter Angélique. Si le merveilleux arthurien est largement mis en valeur, l'humour pointe souvent - ainsi dans l'aventure de Roland qui est tombé dans la rivière des Naïades, les chevaliers venus à son secours remontent de la rivière enchantée «comme des courges», et les métaphores réalistes et comiques contrastent avec des métaphores élevées, empruntées à Pétrarque.

Les plus grands mythes classiques sont réécrits - ainsi le mythe ovidien de Cadmos est amplifié dans des images extraordinaires, dans l'aventure de Mandricard par exemple, où celui-ci doit combattre contre des oiseaux qui sortent des feuilles et des fleurs, détail exacerbé d'Ovide. Le mythe peut être amplifié de façon élevée mais aussi rabaissé de façon grotesque - ainsi le paladin Roland détruit-il le sphinx à coup d'épée car il n'arrive pas à répondre à la question posée, ou bien c'est le cyclope Polyphème qui se demande s'il ne va pas manger Roland car il est «bien dur comme un bon mouton». Boiardo agit à l'égard de sa matière toujours de la même façon - ainsi il reprend à Dante des éléments de sublime merveilleux, notamment ses sonorités ou l'emphase dramatique de la situation, ou bien il le détourne de façon comique en le banalisant, par exemple l'expression de l'arrivée dans une «forêt obscure », incipit de la Comédie, est utilisé pour signaler l'entrée dans l'autre monde, de façon répétitive et formulaire. Il procède donc à une réécriture ludique et humoristique de la matière 
merveilleuse antérieure, ce qui produit sur le lecteur connaisseur un effet de surprise ou d'étonnement.

Plus généralement, ses procédés stylistiques oscillent entre la répétitivité ou banalité et l'étonnement, l'inédit. En effet les analogies vont de la plus simple formule typique des poèmes chevaleresques - «il était plus rapide qu'une flèche »- aux analogies inédites comme «tellement rapide et léger dans sa course / qu'il avait déjà surpassé le vent » (I, 1, 38). Il en est de même pour les hyperboles, la bataille est toujours «cruelle et inouïe», le monstre «horrible et différent», mais parfois certaines visent au surréel: «Et la forêt toute proche et plus lointaine / Vit ses arbres tomber dans cette fureur» (II, 21, 5). Les effets sonores varient entre des séries récurrentes banales et des rimes extrêmement riches et rares, accompagnées d'allitérations et d'assonances pour dire la violence du combat. De même, les répétitions vont de la simple anaphore à la diffraction complexe sur plusieurs vers, dans des chiasmes recherchés qui suscitent la surprise et l'émotion. Ainsi l'on retrouve à tous les niveaux, narratif, intertextuel et stylistique, ce double mouvement de familiarité-banalité et distanciation-dépaysement, principe que nous appelons «émerveillement», issu des «mirabilia» latins et de la «meraviglia» italienne, qui signifie «susciter la surprise et l'admiration par des effets extraordinaires ». Ce principe nous semble pertinent pour cerner la poétique et le style d'un auteur original, redécouvert récemment par la critique ${ }^{1}$ et dont l'écriture mérite d'être étudiée pour la force sonore et évocatrice de ses images et du mélange de ses tons.

1. Voir essentiellement Marco Praloran, Tina Matarrese, Antonia Tissoni Benvenuti et Riccardo Bruscagli. 
Poétique de l'émerveillement dans le «Roland amoureux» de Boiardo

\section{BIBLIOGRAPHIE SÉLECTIVE}

Il Boiardo e la critica contemporanea, Atti del convegno di studi a cura di Giuseppe Anceschi, Firenze, Olschki, 1970.

Il Boiardo e il mondo estense nel Quattrocento, Atti del convegno internazionale di studi a cura di Giuseppe Anceschi e Tina Matarrese, Padova, Antenore, 1998.

BRuSCAGLi Riccardo, Stagioni della civiltà estense, Pisa, Nistri-Lischi, 1983.

MatarRese Tina, » La similitudine nell'Orlando innamorato: il gioco della variazione, dans Schifanoia, 17-18, 1996.

-, «Dalla lirica all'epica: fenomeni di interdiscorvisità nell'Inamoramento de Orlando », dans Gli amorum libri e la lirica del Quattrocento con altri studi boiardeschi, Comune di Scandioan, Centro di studi di M. M. Boiardo, a cura di A. T. Benvenuti, Novara, 2003.

Praloran Marco e Tizi Marco, Narrare in ottave: metrica e stile dell' Innamorato, Pisa, Nistri-Lischi, Pisa, 1988.

PRALORAN Marco, «'La più tremenda cosa posta al mondo'. L'avventura arturiana nell'Innamoramento di Orlando », in La Parola del testo, 1, 1997.

-, «L'alliterazione nell'Inamoramento de Orlando», dans Stilistica e metrica italiana, 1, Firenze, 2001.

-, Il Poema in ottava. Storia linguistica italiana, Roma, Carocci, 2003. SANGIRARDI Giuseppe, Boiardismo ariostesco : presenza e trattamento dell' Orlando innamorato nell' Orlando furioso, Luca, Pacini Fazzi, 1993.

-, «La Commedia di Orlando: dantismo, enfasi e pluritonalità nello stile dell'Orlando innamorato », dans Boiardo e il mondo estense, op. cit., 1998.

Tissoni Benvenuti Antonia, Il Quattrocento settentrionale, Bari, Laterza, 1972.

-, introduction à l'Inamoramento de Orlando, Milano-Napoli, Ricciardi, 1999. 